\title{
Un "mâle" nécessaire pour le programme de la Fédération Wend-Yam
}

Pierre-Joseph Laurent

\section{OpenEdition}

1 Journals

Édition électronique

URL : http://journals.openedition.org/apad/2483

DOI : 10.4000/apad.2483

ISSN : 1950-6929

Éditeur

LIT Verlag

Édition imprimée

Date de publication : 1 décembre 1993

\section{Référence électronique}

Pierre-Joseph Laurent, "Un "mâle" nécessaire pour le programme de la Fédération Wend- Yam », Bulletin de l'APAD [En ligne], 6| 1993, mis en ligne le 10 mars 2008, consulté le 08 septembre 2020. URL : http://journals.openedition.org/apad/2483; DOI : https://doi.org/10.4000/apad.2483

Ce document a été généré automatiquement le 8 septembre 2020

Bulletin de l'APAD 


\title{
Un "mâle" nécessaire pour le programme de la Fédération Wend- Yam
}

\author{
Pierre-Joseph Laurent
}

1 Les associations paysannes, surgissent aujourd'hui de partout, poussées dans le dos par les changements récents de l'environnement économique (le programme d'ajustement structurel), politique (la démocratisation et les mouvements de décongestion décentralisation de l'Etat) et social (l'émergence de nouveaux acteurs et d'autres rapports sociaux) ${ }^{1}$. J.-P. Jacob montre très bien - qu'on déplore ou qu'on en fasse l'apologie - que la multiplicité des organisations paysannes est un fait au Burkina Faso ${ }^{2}$. La nouvelle mode de développement n'a paradoxalement pas toujours un effet positif sur les populations à la base. Nous voudrions dans ces quelques pages montrer que cette politique peut conduire au renforcement d'acteurs qu'il est légitime de nommer "les courtiers locaux du développement" 3.

Il existe quelques associations paysannes qui émanent d'un projet paysan. Elles naquirent, généralement loin des sentiers fréquentés par la coopération au développement. Aujourd'hui courtisées, elles s'étonnent encore de l'intérêt qu'elles suscitent. Sortir de l'ombre, même avec des appuis bienveillants, représente un défi quotidien, pour ces acteurs paysans. Ils doivent intérioriser rapidement les règles d'un nouvel espace-temps et s'y conformer. Promus représentants de la paysannerie, ils seront invités par les bailleurs à des séminaires, des tables rondes, des colloques à l'occasion desquels ils seront amenés à négocier. Ils tenteront de drainer des ressources vers leur base. Ils se heurteront cependant à de multiples problèmes, dont l'analphabétisme. Face à ces lacunes parfois sous-estimées par les partenaires étrangers, des acteurs, dont le rôle consiste précisément à détecter les faiblesses des partenaires-bénéficiaires, veillent. Ces "nouveaux intermédiaires" proposent leurs services sur le terrain de la recherche de financements, à des paysans désorientés : la transaction tourne alors autour du partage de ressources de la coopération au développement. 
3 Il sera ici question d'un "leader paysan" à qui son association, ainsi que certains bailleurs, ont confié le rôle de "courtier de développement aux pieds nus" ${ }^{4}$. La fédération Wend-Yam émane d'un projet paysan. Elle fut fondée le 28 septembre 1985 par une poignée de villageois mossi de Kulkinka de la province d'Oubritenga au Burkina Faso. Des motivations religieuses animaient les premiers adhérents. Elles se transformèrent ensuite, entraînées dans un mouvement de sécularisation, vers une institution de développement, dirigée par des représentants élus ${ }^{5}$. L'association rassemble 58 villages et plus de 5000 membres. Ils se répartissent entre $45,9 \%$ d'animistes, 33,9\% de musulmans, $15,6 \%$ de protestants (évangélistes) et $4,6 \%$ de catholiques. La fédération Wend-Yam juridiquement reconnue (ONG de droit burkinabé), met en œuvre de multiples activités, notamment une banque d'épargne et de crédit pour le développement d'activités d'élevage et l'octroi de matériel agricole, un réseau de banques de céréales et de moulins, l'organisation de périmètres maraîchers, la lutte contre l'érosion des terres agricoles, le reboisement d'espaces communaux, les formations professionnelles, les cours d'alphabétisation, etc. ${ }^{6}$.

Depuis sa création, la fédération est dirigée et administrée par un groupe de douze villageois, élus pour un mandat de deux ans. Il constitue le "bureau provincial", lequel se réunit tous les jeudis. Les membres de ce bureau n'ont pas fréquenté l'école. Seuls cinq animateurs embauchés par leurs soins possèdent une formation de niveau secondaire (deux supérieurs et trois inférieurs). Les animateurs collaborent essentiellement à la mise en œuvre des actions. "Les papiers" demeurent la hantise de la fédération, qui gère néanmoins un budget annuel de 80 millions de FCFA. Ils obtiennent leurs financements auprès de bailleurs rencontrés, à la faveur d'un réseau de relations savamment entretenu, auprès des représentants d'ONG ou des instances de coopération bilatérale installées à la capitale. Entre 1988 et 1991, l'association paysanne fut aidée dans la gestion "des papiers" et plus concrètement dans la rédaction et la présentation des dossiers de projet, par un expatrié ${ }^{7}$. Leur réseau de partenariat s'élargit durant cette période. Mis à part ce soutien significatif, mais ponctuel, la filière de confection des dossiers de projet est généralement la suivante : les responsables de l'association discutent d'abord du bien fondé d'une demande transmise par un groupement villageois adhérant. La demande une fois approuvée, sera portée devant un écrivain public de la capitale à qui le Président dicte de mémoire, le texte du futur projet. Un dactylographe se charge ensuite de la mise en forme du document qui sera présenté enfin au bailleur pressenti.

5 En 1991, face aux difficultés que représente la rédaction des dossiers de projet ainsi que les remaniements successifs du texte rendus nécessaires par les demandes de précisions des bailleurs, la fédération décide d'opter pour la réalisation d'un "programme". L'association entend par là dresser un plan indicatif de ses futures activités. Dans cette perspective, les bailleurs qui accorderaient leur confiance aux gestionnaires paysans, négocieraient entre eux une répartition du financement $\mathrm{du}$ programme. Ils délégueraient ensuite, sans autres formalités, les subsides à l'association, étant bien entendu que le contrôle se ferait a posteriori. La proposition présente l'indéniable avantage de faire l'économie de la pénible phase de rédaction du dossier de projet, vécue par les villageois, comme un véritable parcours du combattant. La démarche fut approuvée à l'Assemblée Générale du mois de juin 1991, qui décida de se lancer dans la réalisation d'un programme quadriennal. Ce travail nécessitera trois années pour aboutir. Le document final a été présenté le 25 juin 1993 à Bruxelles par le 
Président de l'association, à l'occasion d'une table ronde organisée par ses principaux bailleurs. L'ordre du jour de cette rencontre portait cependant sur autre chose: l'assistance technique auprès de la fédération Wend-Yam.

6 L'élaboration du programme quadriennal se déroule en six points. La première étape consiste dans la prise de position de l'Assemblée Générale en faveur du "programme" (voir supra). La seconde étape représente la convocation, au siège de la fédération, des délégués des 58 groupements villageois membres, afin de leur expliquer les objectifs de la démarche. La rencontre dura deux jours. Afin de faciliter la réalisation du programme, l'agence de coopération qui avait suggéré la démarche à l'association délégua pendant trois jours un consultant. De l'avis des intéressés, sa méthode, consiste à "questionner perpétuellement les paysans sur les motifs de leurs activités programmées".

7 La troisième étape se compose de la visite aux 58 groupements villageois, aux animateurs et aux paysans engagés dans les instances de la fédération (ces derniers sont bénévoles) afin de réfléchir avec les membres à la réalisation de deux listes. La première reprend à la fois les activités réalisées par le groupement et celles qu'ils entreprendront. La seconde répertorie les investissements à réaliser dans le village et pour lesquels des subsides sont demandés. La quatrième étape consiste dans l'extension de la démarche précédente, à la structure institutionnelle de la fédération Wend-Yam. Le Bureau Provincial qui représente l'organe exécutif, ainsi que les six Bureaux Départementaux ont mené une réflexion sur les modalités de leur viabilité.

8 La cinquième étape comporte une rencontre de coordination. Elle rassemble l'ensemble des personnes qui visitèrent les villages. La rencontre eut lieu au siège de la Fédération du 06.10.92 au 09.10.92. L'ensemble de l'information recueillie auprès des villageois est trié et synthétisé. Les débats portent sur chaque activité importante, ainsi que sur l'analyse de la pertinence des demandes fournies par chaque village. L'assemblée dresse ensuite une typologie composée de cinq catégories d'activités: sociales: forages, pharmacies, dispensaire, maternité, P.M.I., logement d'infirmier, banques de céréales, magasin de stockage, fonds de roulements, château d'eau, abattoir, panneaux solaires, route, pirogues, vivres; éducation et formation: alphabétisation, formations professionnelles, école, logement de l'instituteur, formation des responsables de la fédération, garderie d'enfants; gestion du terroir: diguettes, reboisement, barrage, retenues d'eau, "boullis", traitement des ravines, fosses fumières, foyers améliorés ; économiques : savonnerie, tissage, station d'essence, apiculture, moulins à céréales, motopompes, maraîchage, riziculture, tracteurs, banques d'épargne et de crédit, crédit boutiques villageoises, crédit matériel agricole et pour l'élevage, traitement phytosanitaire ; viabilité de l'institution Wend-Yam : centre de formation paysanne, un centre artisanal, un programme général de formation, la commercialisation d'essence, le commerce de ciment avec la Côte d'Ivoire, le commerce de produits vivriers avec la Côte d'Ivoire, autocars, tracteurs, camions, énergie solaire, route, crédit pour une banque rurale, soins de santé pour les membres, fonds d'urgence en cas de sinistre, informatisation ${ }^{8}$. Ces données représentent la base du plan quadriennal.

9 À la fin de la rencontre, le problème de la rédaction du document se pose car aucun d'entre eux ne peut réellement assumer cette tâche. Voici comment le Président voit les choses :

«Cela fait plus d'un an que nous avons commencé le programme. Il sera comme un

livre, un bon livre qui voyagera en Europe et en Afrique. Dans ce travail, chacun y 
trouvera son intérêt. Si des gens nous posent des questions, nous devons tous être capables d'y répondre. Les trois races sont ici : Blanche, Peul et Mossi, techniciens et paysans, nous devons faire l'effort d'expliquer notre programme ». orientations de la fédération. Un plus grand document présentera la fédération WendYam et son plan quadriennal. Le choix des membres de ce comité est délicat. Cinq personnes seront finalement choisies: l'animateur, adjoint du président (études secondaires supérieures accomplies); le directeur provincial de l'action coopérative (spécialiste en animation rurale et ami du Président); un expatrié (agronome, collaborateur occasionnel de la fédération); un sociologue tchadien (ayant réalisé son stage de fin d'études auprès de l'association) et le Président de la fédération (cultivateur et pasteur protestant). Le conseiller de la fédération rappela au comité de rédaction les déconvenues passées (comme par exemple les sommes englouties dans la rédaction d'un dossier de projet alors que des villages se sont cotisés pour payer en vain les services d'un technicien). Il résuma sa pensée par un dicton :

«Celui qui a vu les abeilles c'est bien, mais c'est toutefois celui qui a construit

l'échelle qui ira chercher le miel» terminé. En 8 pages, il présente les nouvelles orientations prises par l'association paysanne. Il s'articule autour de six points :

- le renforcement de l'autonomie de la structure institutionnelle de la Fédération ;

- le renforcement de la structure de communication de la fédération ;

- le développement d'actions, aux bénéfices individualisables et collectifs ;

- le renforcement et la distinction précise entre des activités à caractère économique et social ;

- l'accentuation des formations professionnelles, de l'alphabétisation et des formations pour les paysans formateurs ;

- le rôle des femmes au sein de l'association.

entisation du document de présentation du programme demande encore un an de travail. Il se clôture à l'occasion du premier voyage en Europe, en juin 1993, du Président de l'association. Le document, finalement intitulé "Plan triennal de 1994-1996", comprend deux parties. La première compte 12 pages et présente dans une formulation assez guindée la fédération Wend-Yam. La seconde partie décrit le programme à l'aide d'un tableau s'étendant sur 6 pages. Les actions retenues y sont ventilées entre 47 rubriques, elles-mêmes réparties sur trois années. Le montant total du programme s'élève à deux milliards trois cent quatre vingt FCFA.

Le Président de la fédération Wend-Yam se présente le 23 juin 1993 à la table ronde des bailleurs à Bruxelles à laquelle assistent trois ONG européennes. Quelques jours avant cette date, de nombreux paysans d'Oubritenga se pressent à l'aéroport pour saluer leur ambassadeur. Le voyage préparé de longue date revêt une importance particulière : les villageois confiants dans leur représentant espèrent des promesses de financements. La visite se soldera toutefois sur un constat d'échec. Le Président commente ainsi son voyage en Europe et sa rencontre avec "le monde de la coopération".

"Les bailleurs suivent leurs objectifs et les paysans les aident à les remplir. Les

bailleurs mènent leur politique pour obtenir du pouvoir. C'est bien clair. Pourtant 
dans leurs papiers ils indiquent qu'ils aident les paysans. Nous pouvons connaître des réussites, mais ce n'est pas cette réussite là qui les intéressent".

Le séjour du Président en Europe fut une accumulation d'incompréhensions. La communication entre les parties ne put jamais réellement s'établir. Ni le document programme, ni les arguments du Président n'ont convaincu. La communication, sans intermédiaires (sans médiateurs ou encore sans "courtier du développement ${ }^{21}$ ) fut, dans ce cas, un cuisant échec. Voici comment le Président de la fédération Wend-Yam analyse la situation à la fin de son séjour.

Appréciations générales

"Nous nous sommes fatigués pour réaliser le plan triennal. Cela fait trois ans que nous y réfléchissons, mais ils ne l'ont pas compris. Tu prends trois ans pour parler avec les villages, pour leur ouvrir les yeux et pour mener des études avec eux. Le document est finalement écrit, mais les bailleurs nous disent qu'il représente trop d'argent. Pourtant, même si les paysans reçoivent cet argent, cela ne suffira pas à apaiser leurs problèmes. Les bailleurs disent que la fédération Wend-Yam gère 80 millions et que c'est déjà beaucoup. Ils disent cela parce que nous sommes paysans".

Découragement

"Maintenant je suis dans un cachot et je sais pas où se trouve la porte pour sortir. C'est comme si j'étais reçu par un ami qui, content de me voir, ne me donnerait cependant, ni à boire, ni à manger, ni une chambre. Je suis à sa porte avec mon sac et il ne me donne pas de chaise".

Énoncé du problème

"Les bailleurs doivent accepter les paysans. Je veux dire, accepter ce qu'ils ont besoin. Les bailleurs nous ont proposé de faire un programme. Maintenant que le programme existe, ils disent que notre étude est insuffisante. Ils pensent que la tête des paysans n'est pas valable. Nous n'avons pas fait d'études. Je n'ai pas fréquenté l'école et je n'ai personne "de haut" à qui demander des avis. Je pense que pour la somme (c'est-à-dire le montant prévu pour le financement du programme), les bailleurs voudraient 200 pages, peut-être même 300 pages. Si un intellectuel collaborait avec nous, il aurait pu écrire un livre. Pourtant j'ai écouté leur conseil. Pour cela, j'ai suivi leur chemin (c'est-à-dire, l'exemple des projets financés par ses bailleurs). J'ai visité leur travail au Burkina, au Mali et au Sénégal et je dois dire que si ce n'est pas la fédération Wend-Yam, les autres organisations n'écrivent pas eux mêmes leurs dossiers".

Objectif du plan triennal

"Dans le petit document, intitulé "les nouvelles orientations", nous avons expliqué aux bailleurs ce que nous espérions par la réalisation du programme. Pourquoi les bailleurs ne nous ont pas répondu ? Je ne sais pas si ce que nous faisons est bien ou mal. Nous avons détaillé, dans le document de présentation du plan triennal, les activités demandées pour chaque village. Nous pensions que dans cette liste chaque bailleur choisirait ce qu'il voudrait bien financer. Mais ce n'est pas cela qu'ils veulent. Ils veulent un budget précis, pour des activités précises, comme un forage ou un moulin... Mais si c'est cela, je peux payer quelqu'un pour leur faire un dossier de projet. Le problème, il est là. Je pensais que les bailleurs nous avaient compris puisqu'ils avaient dit qu'ils voulaient appuyer la fédération Wend-Yam".

"Les bailleurs doivent laisser les associations paysannes avec les problèmes de dossiers. Si nous savions écrire des projets, nous ne serions pas paysans. Ils savent que je suis analphabète. Ils doivent donc savoir que cela veut dire que je ne sais pas écrire. Le système de la coopération ne marche pas. Les bailleurs ne peuvent pas nous suivre. Ils travaillent comme avant, comme il y a trente ans. C'est comme s'ils avaient mis une cale sur mon vélo. Maintenant, je dois mettre le plan triennal dans mon tiroir et continuer à écrire des projets comme avant.

La confiance 
"Les bailleurs, s'ils acceptent de nous financer, doivent nous faire confiance. La fédération Wend-Yam, avec le plan triennal, propose un nouveau système. Jamais une association paysanne n'a rédigé son programme elle même. Cependant, pour que cela fonctionne, la confiance doit régner. Les bailleurs pourraient nous avancer l'argent. À la moitié du travail, ils devraient réaliser une évaluation et ainsi de suite. Ils pourraient ainsi se rendre compte de notre travail. Les grandes associations, elles, montrent ce qui fonctionne; les bailleurs veulent cela : un bon barrage par exemple. Ils discutent un peu, ensuite il y a des danses et enfin chacun rentre chez lui. Comme cela, je ne vois pas tellement comment le développement arrivera. Non, les bailleurs doivent venir voir le travail chaque année, afin de vérifier s'il a été réalisé. Pour cela, j'aimerais que les bailleurs contrôlent les réalisations et la comptabilité. Ils ne viendraient pas pour recevoir une chèvre ou pour les danses; ils dureraient, c'est-à-dire qu'ils prendraient le temps de visiter chaque village, afin d'écouter les gens parler de leurs réalisations".

"Les bailleurs doivent surtout nous laisser tranquilles avec les problèmes de rédaction des dossiers de projets. Ils ne devraient pas nous obliger à les écrire nous même. S'ils veulent que les paysans se développent, ils doivent vraiment travailler avec nous et pour cela il ne faut pas nous fatiguer avec ces problèmes. Les bailleurs viendraient ici pour écrire leur dossier. À "occasion de cette visite ils verraient notre travail et sur cette base ils décideraient de nous financer. Ce n'est pas sur des explications contenues dans un dossier que les bailleurs doivent se décider".

Projet et programme

"Si on veut un atelier de soudure par exemple, on l'indique dans un dossier. On calcule le prix et on explique que "atelier servira à ceci et à cela. Si un bailleur passe et qu'il veut le financer, il prend le dossier. C'est cela un projet. Le programme c'est différent. Les bailleurs viennent et ils voient des choses : c'est notre témoignage. Ils vont alors sur cette base dire, voilà, j'accepte de faire cela avec vous. C'est alors eux, qui réalisent le dossier dans lequel ils expliquent tout afin de faire comprendre la situation à leur gouvernement".

De retour chez lui, le Président plaça comme annoncé, le plan triennal dans le tiroir de son bureau. Toutefois, avant de rentrer au Burkina Faso, il prit soin d'acheter un ordinateur. Peut-on y voir la volonté de l'appropriation, sinon de l'écriture, de la possibilité de la présentation de documents de qualité.

Confrontée à l'échec du programme, la fédération Wend-Yam, après trois années d'interruption, renoue avec la réalisation des dossiers de projets. Un dossier technique leur coûte de 75.000 à 250.000 francs CFA Cette somme représente le prix à payer pour s'adjoindre les services d'un "courtier en développement". Deux filières s'ouvrent à eux pour le recrutement de ces acteurs. Premièrement, des agents des services techniques provinciaux: dans ce cas, le produit fini se révèle de faible qualité, mais leur rétribution reste modeste. Ces dossiers sont destinés à des bailleurs nationaux. Deuxièmement, les consultants nationaux auprès des agences de coopération internationales ou les cadres des ONG. Leurs honoraires sont plus élevés mais, mieux informés, ils se montrent efficaces auprès des institutions de développement.

Pour reprendre les termes de l'article de J.-P. Olivier de Sardan et de Th. Bierschenk, le Président de l'association paysanne, rapidement promu "courtier du développement aux pieds nus" ${ }^{10}$ par certains bailleurs, n'a toutefois pas été reconnu comme tel, par d'autres. La tentative originale de communication sans intermédiaire entre les bailleurs et les bénéficiaires n'a pas (ou pas encore) fonctionné. Les interlocuteurs locaux, s'ils prennent aujourd'hui de l'importance recourent toujours, dans notre cas, aux services de "courtiers du développement" expérimentés, c'est-à-dire capables de traduire les besoins des uns en projets pour les autres, moyennant une rente à négocier. 


\section{BIBLIOGRAPHIE}

Gentil D., Plan d'ajustement structurel, démocratisation et organisations paysannes, Paris, Journées d'études IRAM, septembre 1993.

Jacob J.-P., "Imputabilité, redondance et pouvoir. Réflexions sur la multiplicité des organisations paysannes au Burkina Faso", in Jacob J.-P. et Lavigne Delville Ph., Les associations paysannes, Paris, Karthala, à paraître.

Laurent P.-J., "Intensification agricole, prosélytisme religieux et association paysanne. Le rôle des 'Assemblées de Dieux' dans l'émergence de la Fédération Wend-Yam", in Jacob J.-P. et Lavigne Delville Ph., Les associations paysannes, Paris, Karthala, à paraître.

Olivier de Sardan J.-P. et Bierschenk Th. "Les courtiers locaux du développement", Bulletin $A P A D, n^{\circ} 5$ Marseille, juin 93, pp. 71-76.

Nouvelles Orientations. Programme Quadriennal 1993-1996, Ziniaré, Fédération Wend-Yam, novembre 1992,8 p.

Programme triennal de la fédération Wend-Yam, Ziniaré, juin 1993, 14 p.

Rapport d'activités de la Fédération Wend-Yam, Juillet 1993.

\section{NOTES}

1.Voir à ce propos D. Gentil, Plan d'ajustement structurel, démocratisation et organisations paysannes, Journées d'études IRAM, Paris, septembre 1993, p. 1.

2.J.-P. Jacob, "Imputabilité, redondance et pouvoir. Réflexions sur la multiplicité des organisations paysannes au Burkina Faso", in J.-P. Jacob et Ph. Lavigne Delville, Les associations paysannes, Paris, Karthala, à paraître.

3.Voir à ce propos J.-P. Olivier de Sardan et Th. Bierschenk, "Les courtiers locaux du développement", Bulletin $n^{\circ} 5$ APAD, Marseille, juin 93, p. 71.

4.J.-P. Olivier de Sardan et Th. Bierschenk, op. cit., p. 73.

5.Voir P.-J. Laurent, "Intensification agricole, prosélytisme religieux et association paysanne. Le rôle des 'Assemblées de Dieux' dans l'émergence de la Fédération Wend-Yam", in J.-P. Jacob \& Ph. Lavigne Delville, Les associations paysannes. Paris, Karthala, à paraître.

6.Voir Rapport d'activités de la Fédérarion Wend-Yam, Juillet 1993.

7.En l'occurrence ici, moi-même : médiateur par vocation.

8. Nouvelles Orientations. Programme Quadriennal - 1996, Ziniaré, Fédération Wend-Yam, novembre 1992, p. 7 et 8.

9.J.-P. Olivier de Sardan \& Th. Bierschenk, op. cit., p. 73.

10.J.-P. Olivier de Sardan \& Th. Bierschenk, op. cit. p. 73. 